Swarthmore College

Works

6-12-2014

\title{
Columnar Molecular Aggregation In The Aqueous Solutions Of Disodium Cromoglycate
}

D. M. Agra-Kooijman

G. Singh

A. Lorenz

Peter J. Collings

Swarthmore College, pcollin1@swarthmore.edu

H.-S. Kitzerow

See next page for additional authors

Follow this and additional works at: https://works.swarthmore.edu/fac-physics

Part of the Physics Commons

Let us know how access to these works benefits you

\section{Recommended Citation}

D. M. Agra-Kooijman, G. Singh, A. Lorenz, Peter J. Collings, H.-S. Kitzerow, and S. Kumar. (2014).

"Columnar Molecular Aggregation In The Aqueous Solutions Of Disodium Cromoglycate". Physical Review E. Volume 89, Issue 6. DOI: 10.1103/PhysRevE.89.062504

https://works.swarthmore.edu/fac-physics/128

This work is brought to you for free by Swarthmore College Libraries' Works. It has been accepted for inclusion in Physics \& Astronomy Faculty Works by an authorized administrator of Works. For more information, please contact myworks@swarthmore.edu. 
Authors

D. M. Agra-Kooijman, G. Singh, A. Lorenz, Peter J. Collings, H.-S. Kitzerow, and S. Kumar 


\title{
Columnar molecular aggregation in the aqueous solutions of disodium cromoglycate
}

\author{
Dena M. Agra-Kooijman, ${ }^{1}$ Gautam Singh, ${ }^{1}$ Alexander Lorenz, ${ }^{2}$ Peter J. Collings, ${ }^{3}$ Heinz-S. Kitzerow, ${ }^{4}$ and Satyendra Kumar ${ }^{1},{ }^{*}$ \\ ${ }^{1}$ Department of Physics, Kent State University, Kent, Ohio 44242, USA \\ ${ }^{2}$ Department of Chemistry, Berlin Institute of Technology, Strasse des 17. Juni 124, 10623 Berlin, Germany \\ ${ }^{3}$ Department of Physics \& Astronomy, Swarthmore College, Swarthmore, Pennsylvania 19081, USA \\ ${ }^{4}$ Department of Chemistry, University of Paderborn, Warburger Strasse 100, 33098 Paderborn, Germany
}

(Received 13 February 2014; published 12 June 2014)

\begin{abstract}
Stack, chimneylike, and threadlike assemblies have previously been proposed for the structure of disodium cromoglycate (DSCG) aggregates in aqueous solutions. The results of the synchrotron x-ray scattering investigations reported here reveal the formation of simple columnar assemblies with $\pi-\pi$ stacking at a separation of $3.4 \AA$ between the DSCG molecules. Lateral separation between the assemblies is concentration and temperature dependent, varying from $\sim 35$ to $42 \AA$ in the orientationally ordered nematic $(N)$ phase and from 27 to $32 \AA$ in the columnar or middle $(M)$ phase having long range lateral positional order. The assemblies' length depends on concentration and consists of $\sim 23$ molecules in the $N$ phase, becoming three to ten times larger in the $M$ phase. The scission energy is concentration dependent in the $N$ phase with values $\sim 7.19 \pm 0.14$ $k_{B} T(15 \mathrm{wt} \%), 2.73 \pm 0.4 k_{B} T(20 \mathrm{wt} \%)$, and $3.05 \pm 0.2 k_{B} T(25 \mathrm{wt} \%)$. Solutions of all concentrations undergo a spinodal decomposition at temperatures above $\sim 40^{\circ} \mathrm{C}$, resulting in DSCG-rich regions with the $M$ phase and water-rich regions in the $N$ and isotropic phases.
\end{abstract}

DOI: 10.1103/PhysRevE.89.062504

PACS number(s): 61.30.Eb, 61.30.Cz, 61.05.cf

Chromonic liquid crystals (CLCs) are comprised of orientationally and sometimes positionally ordered anisotropic assemblies of plank-shape dye molecules in aqueous solutions. Unlike the better-known lyotropic liquid crystals, there is no micelle formation in CLCs even though molecular assemblies form as a consequence of their hydrophobic aromatic flat core and ionic groups at the outer edges of the molecules. Dye molecules undergo $\pi-\pi$ stacking to form assemblies, the size and density of which depend on concentration, temperature, and the $p \mathrm{H}$ value of the solution. These assemblies then develop order and form the nematic $(N)$ and columnar or middle $(M)$ liquid crystal (LC) phases. Chromonic solutions have traditionally been used in pharmaceuticals [1,2], food coloring [3], coatable polarizers, and optical compensators [4] suitable for flexible optical and electronic applications [5]. In recent years, researchers have explored several new applications [6], for example, organic field effect transistors, optical switches, reprographics, solar energy collectors, and detection [7] of biological and chemical agents.

Ever since Cox et al. [1] reported that hydrated disodium cromoglycate (DSCG) forms columnar assemblies and exhibits the birefringent $N$ and $M$ liquid crystal phases, this system has been of continued interest [8,9] to scientists. X-ray diffraction studies [9] of this system concluded that the $N$ phase is comprised of orientationally ordered assemblies [Fig. 1(a)], which were hexagonally packed in the $M$ phase. Lydon [10] later proposed the molecular assembly in this system to be (chimneylike) hollow-square columns in which four molecules were linked by electrostatic salt bridges [Fig. 1(b)].

Although Lydon retracted [11] the hollow-square model in 1984 , it was later reported to be consistent with the nuclear magnetic resonance and x-ray results of Goldfarb et al. [12]. Interestingly, evidence of a similar hollow brickwork

*Corresponding author: skumar@kent.edu (chimney) structure was later reported [13-15] for a different chromonic dye. Several other dyes, such as C. I. Direct Blue 67 [15], Sunset Yellow FCF [16], or Violet 20 [17] also form assemblies in water. Reviews by Lydon [18] and Collings [19] provide details of chromonic systems.

Recently, Wu et al. [20] proposed a very different model based on small-angle neutron scattering results. They proposed "threadlike" assemblies [Fig. 1(c)] of molecules connected by salt bridges stacked on top of aromatic groups. The threads are believed to then self-organize to form the $N$ and $M$ phases.

In order to determine which of the three models best describes the DSCG system and to gain deeper understanding of the mesophase formation, aqueous solutions of DSCG at three concentrations $(15,20$, and 25 wt $\%)$ were selected to access the $N$ and $M$ phases in different parts of the schematic phase diagram [9,19], Fig. 2(a). Their structures were investigated using synchrotron $\mathrm{x}$-ray radiation as a function of temperature. DSCG was purchased from Spectrum Chemical with the commercial name cromolyn sodium in powder form and also used in a previous study [21]. Although the manufacturer specified its purity at $98 \%$, no trace of impurity could be seen in thin layer chromatography. Aqueous solutions of DSCG were prepared without further purification using de-ionized water (HPLC grade), mixed in sealed vials, and allowed to equilibrate for $24 \mathrm{~h}$. The nature of the phases and transition temperatures were determined by filling the solutions in microscope slides, sealing with epoxy to prevent water evaporation, and placing them inside a Mettler FP90 hot stage for temperature control with a temperature stability of $\pm 0.1{ }^{\circ} \mathrm{C}$. Optical textures of mesophases were examined under a polarizing microscope (Olympus BX51) both upon heating and cooling. It should be noted that the phase diagrams reported in the literature vary significantly due to different levels of purity from one batch of DSCG to another.

For x-ray diffraction, DSCG solutions were filled in 1.5$\mathrm{mm}$-diameter quartz capillaries in the isotropic phase, flame sealed, and placed inside an INSTEC (model HS450, $\Delta T \pm$ 


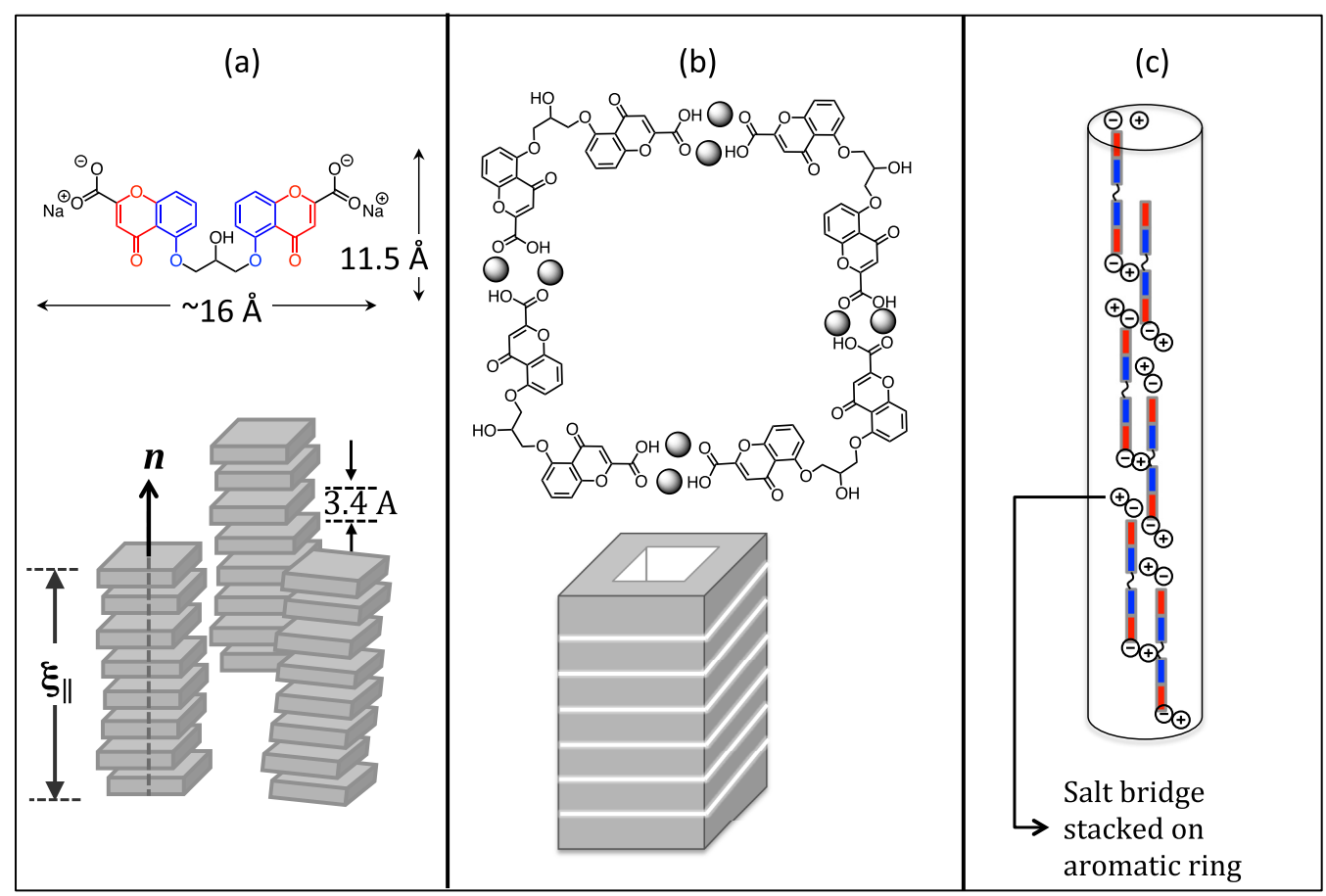

FIG. 1. (Color online) DSCG molecular assembly models by (a) Hartshorne and Woodard [9]; (b) Lydon [10], and (c) Wu [20] et al. The blue and red bars in (c) represent edge-on views of the aromatic rings in the DSCG molecular structure in (a).

$0.1^{\circ} \mathrm{C}$ ) oven with a pair of built-in permanent rare-earth magnets to orient the director. The permanent magnets produced a field of approximately $2.5 \mathrm{kG}$ at the sample. Experiments were performed several (5) days after capillary preparation at sector X6B of the National Synchrotron Light Source using a wavelength of $\lambda=1.03258 \AA$ and a Princeton Instruments two-dimensional detector SCX:4300/1-165. The sample to detector distance was $255 \mathrm{~mm}$. The data were corrected for background scattering from an empty capillary, calibrated against a silver behenate standard, and analyzed using the software FIT2D [22]. All x-ray measurements were made on cooling from the isotropic phase with 10 min of equilibration at each temperature. Experimental details of measurements performed at beamline ID02 of the European Synchrotron
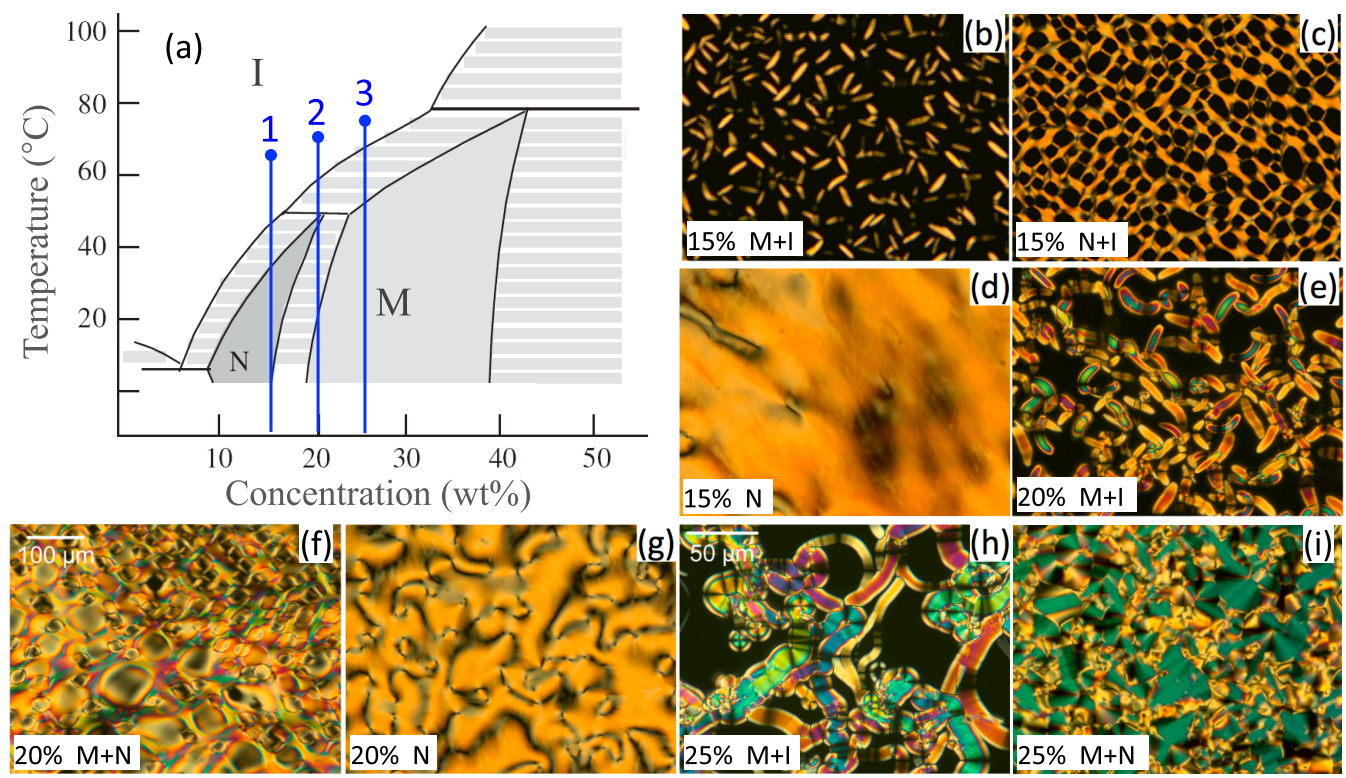

$15 \% \mathrm{~N}$

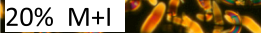

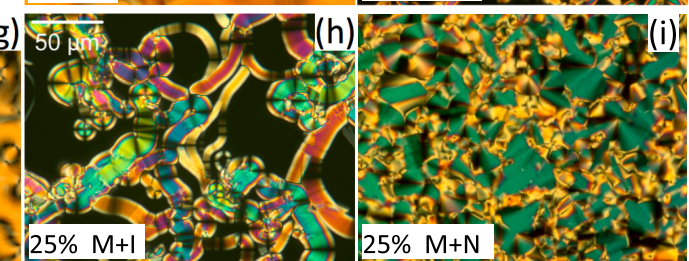

FIG. 2. (Color online) (a) Schematic phase diagram of DSCG reproduced from Ref. [18] with permission from Taylor \& Francis Ltd. The lines marked as 1,2 , and 3 represent the 15,20 , and $25 \mathrm{wt} \%$ concentrations, respectively. The (representative) optical textures correspond to the (b) coexisting $M+I$ phases at $43.8^{\circ} \mathrm{C}$, (c) $N+I$ phases at $38^{\circ} \mathrm{C}$, and (d) the $N$ phase at $23^{\circ} \mathrm{C}$ of the 15 wt $\%$ sample; the (e) $M+I$ region at $44{ }^{\circ} \mathrm{C}$, (f) $M+N$ phases at $38^{\circ} \mathrm{C}$, and (g) the $N$ phase at $25{ }^{\circ} \mathrm{C}$ of the $20 \mathrm{wt} \%$ sample; (h) the biphasic $M+I$ region at $57,{ }^{\circ} \mathrm{C}$ and (i) the $M+N$ phases at $40^{\circ} \mathrm{C}$ of the $25 \mathrm{wt} \%$ sample. 
Radiation Facility (ESRF) are described in Refs. [23,24]. During these measurements, the sample temperature was changed continuously but slowly $\left(1.0^{\circ} \mathrm{C} / \mathrm{min}\right)$. Three regions of the sample at different heights in the capillary were probed to confirm that concentration gradients did not develop over time. The results from different synchrotron experiments performed about 10 months apart on some of the same samples were reproducible, confirming their chemical and thermodynamic stability.

Optical observations reveal characteristic textures of the $N$ and $M$ phases, and biphasic regions [8,19] of $N+I, M+I$, and $M+N$ phases as shown in Fig. 2. The relative volume proportions of the coexisting phases evolve with temperature. The three samples show an $I+M$ coexistence [Figs. 2(b), 2(e), and $2(\mathrm{~h})]$ near the clearing point. The birefringent areas in these textures are elongated with striations, or stripes with broken fan shapes. Although surprising, the presence of the $M$ phase in this region was confirmed with x-ray diffraction, as discussed later in this paper. At lower temperatures, the $15 \mathrm{wt} \%$ mixture reveals an $N+I$ [Fig. 2(c)] biphasic region above the $N$ phase with schlieren texture [Fig. 2(d)]. Regions of $M+N$ coexistence and the $N$ phase are respectively shown [Fig. 2(f) and 2(g)] for the $20 \mathrm{wt} \%$ sample. The coexistence region at the $N$ to $I$ transition is composed of two or more of the $I, N$, and $M$ phases. The 25 wt $\%$ sample exhibits an $M+N$ region [Fig. 2(i)] before transforming to the pure $M$ phase.

The diffraction pattern of the $N$ phase of the $15 \mathrm{wt} \%$ sample at $27.7^{\circ} \mathrm{C}$ consists of five peaks and is shown in Fig. 3(a). Logarithmic plots of the intensity vs $q$ on the right-hand side show the peaks more clearly. The smallest-angle peak corresponds to the lateral distance $\left(d_{1}=44.5 \AA\right)$ between the columns while the wide-angle reflection arises from the characteristic separation of $3.4 \AA$ for $\pi-\pi$ stacking between DSCG molecules within the assemblies. The reflection $d_{1}$

(a)

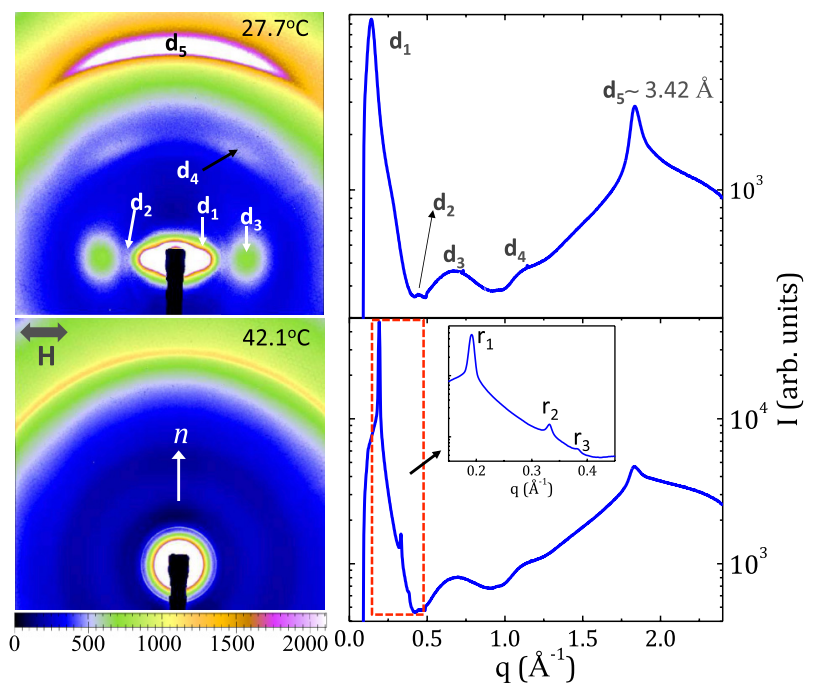

FIG. 3. (Color online) Diffraction patterns for $15 \mathrm{wt} \%$ of DSCG at (a) $T=27.7^{\circ} \mathrm{C}$ and (b) $T=42.1{ }^{\circ} \mathrm{C}$. The corresponding integrated intensity profiles are shown in the right panels. The length scales corresponding to reflections shown in the inset of (b) are $r_{1}=32.8 \AA$, $r_{2}=18.9 \AA$, and $r_{3}=16.4 \AA$, corresponding to peak ratios $1, \sqrt{3}$, and 2 , respectively. appears in the horizontal direction parallel to the magnetic field H. The columns are oriented perpendicular to the applied field. The mutually orthogonal orientation of the $d_{1}$ and $d_{5}=3.4$ $\AA$ peaks establishes that the assemblies consist of molecules that stack on top of one another resembling a deck of cards, depicted in Fig. 1(a), where the nematic director $\boldsymbol{n}$ lies parallel to the long axis of the assemblies. Three weak intermediate peaks at $d_{2}=14.3 \AA, d_{3}=9.4 \AA$, and $d_{4}=5.6 \AA$ are also present [Fig. 3(a)]. These peaks are diffuse and independent of temperature, concentration, and the LC phase. They likely arise from the molecular structure of DSCG. Based on their orientation, low intensity, and relative sharpness, we believe that the peak $d_{2}$ arises from an adsorbed ordered film of DSCG on the capillary walls. The length $14.3 \AA$ corresponds to the largest dimension of the chromone moiety (i.e., half of the molecule) confined in a plane parallel to the magnetic field. The $d_{3}$ peaks are also along $\mathbf{H}$ and appear to correspond to the average width of DSCG molecules inside the assemblies. The diffuse $d_{4}$ reflections, oriented at $\sim 15^{\circ}$ on either side of $\boldsymbol{n}$, arise from the flexible [-O-C-OH-C-O-] segments linking the two flat chromone moieties which are not coplanar and are shifted along the assembly axis, thus orienting the linkage segments at $\pm 15^{\circ}$. It should be noted, however, that the position and the width of the small-angle peak and the width of the $3.4 \AA$ peak depend on temperature and concentration. Full width at half maximum (FWHM) of these peaks allows us to calculate the average number of dye molecules (i.e., the degree of assembly) in a column and the extent over which the hexagonal order in the $M$ phase persists.

In the biphasic region at $T=42.1^{\circ} \mathrm{C}$, the coexistence of the $M$ and $I$ phases is evident from the simultaneous presence of the sharp and diffuse rings shown in Fig. 3(b) and the accompanying intensity vs $q$ plot. For example, the peak $r_{1}$ at $32.8 \AA$ is comprised of a broad and a condensed peak (also shown in Fig. 4) corresponding to the $I$ and $M$ phases, respectively. Additional peaks, $r_{2}$ and $r_{3}$, at 18.9 and $16.4 \AA$, bear the ratios of $\sqrt{3}$ and 2 with respect to the first peak,

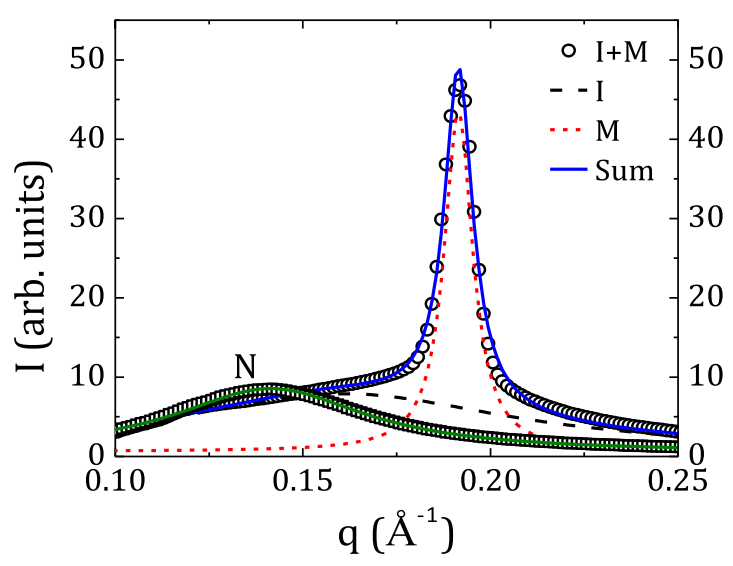

FIG. 4. (Color online) X-ray intensity vs $q$ profiles for the first peak in the $N$ (squares) and $M+I$ (circles) regions of the $15 \%$ sample at $27.7^{\circ} \mathrm{C}$ and $42.1^{\circ} \mathrm{C}$, respectively. The solid lines are Lorentzian fits to data in the $N$ (green squares) phase and the $M+I$ (blue circles) phases. The two fits corresponding to the $I$ (black dashed line) and the $M$ (red dotted line) phases. Their sum fits the $M+I$ data, confirming coexistence of the two phases. 


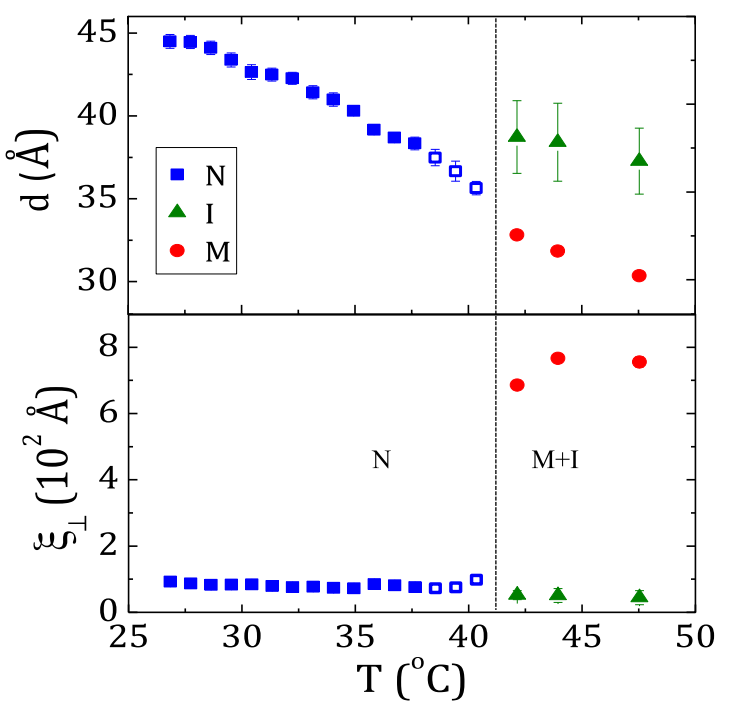

FIG. 5. (Color online) Temperature dependence of the $d$ spacing and positional correlation length $\xi_{\perp}$ for $15 \mathrm{wt} \%$ DSCG.

indicating hexagonal packing of the assemblies in the $M$ phase. In this region, a sum of two Lorentzians is used to obtain a good fit to the composite peak as shown in Fig. 4 confirming the presence of $I$ and $M$ phases below the isotropic phase.

Temperature dependence of $d$ and the positional order correlation length, $\xi_{\perp}$, transverse to the director calculated from the FWHM of the primary peak of the $15 \mathrm{wt} \%$ sample, are plotted in Fig. 5. In the $N$ phase, the lateral distance between the stacks decreases from $\sim 45 \AA$ at $27^{\circ} \mathrm{C}$ to $\sim 35 \AA$ at $40^{\circ} \mathrm{C}$ before the system enters the $M+I$ region. It should be pointed out that the $N$ and $I$ peaks appear at the same position and have comparable widths. At higher $N$ temperatures, it becomes difficult to determine if the measured small-angle peak arises from a single phase or a mixture of the $N$ and $I$ phases. The last three points (open squares) shown in the $N$ phase are in the $I+N$ region identified on the basis of optical textures. Above $\sim 41{ }^{\circ} \mathrm{C}$, the value of $d$ calculated from the $M$ peak further decreases to $\sim 30 \AA$. The FWHM of the lowest- and the largest- $q$ peaks are used to calculate the distances over which the inter- and intracolumn positional order correlations extend. The correlation lengths perpendicular $\left(\xi_{\perp}\right)$ and parallel $\left(\xi_{\|}\right)$to the stacking direction are defined as $2 \pi$ divided by the corresponding FWHM. In the $N$ phase, $\xi_{\perp}(\sim 100 \AA)$ extends roughly over three columns. In the $M+I$ region, $\xi_{\perp}$ increases to $750-800 \AA$ ( $\sim 27$ columns) in the regions of the $M$ phase and becomes $<100 \AA$ in the $I$ phase regions. The optically inferred phase sequence for the $15 \mathrm{wt} \%$ [Figs. 2(b)-2(d)] sample, i.e., $I \rightarrow(M+I) \rightarrow(N+I) \rightarrow N$ is consistent with x-ray results.

Similar results were obtained for the $20 \mathrm{wt} \%$ sample, except for an additional biphasic $N+M$ region which appears on cooling. The XRD pattern, Fig. $6\left(\right.$ a), at $44.8^{\circ} \mathrm{C}$ shows slightly oriented $39.0 \AA$ and $3.4 \AA$ peaks in the $N$ phase. The length scale of $22.7 \AA$ calculated from the second reflection (indicated by the red arrow) corresponds to $\sqrt{3} \times d$ for the primary peak, consistent with a hexagonal structure of the $M$ phase. A sum of two Lorentzians was needed to fit the composite small-angle peak as depicted in Fig. 6(b). The correlation length calculated from the broad $N$ peak is $<100 \AA$ ( $\sim$ three columns) while
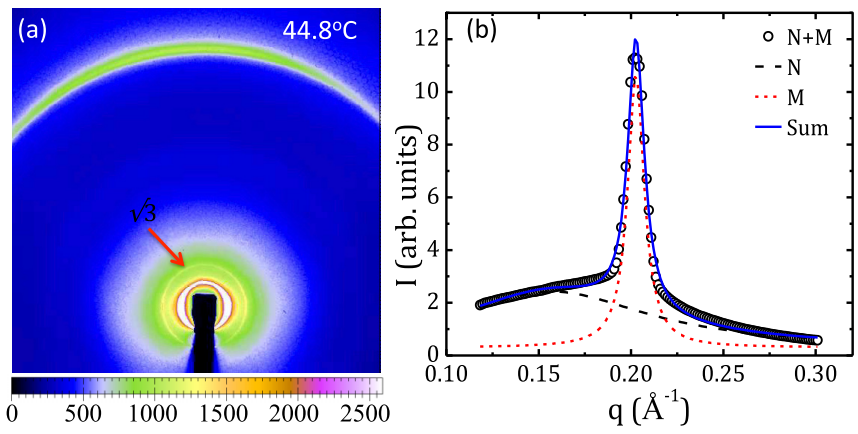

FIG. 6. (Color online) (a) X-ray diffraction pattern for $20 \mathrm{wt} \%$ DSCG in the two-phase regime $N+M$ at $T=44.8^{\circ} \mathrm{C}$ and (b) the corresponding intensity profiles. The blue solid line is the sum of the Lorentzian fits corresponding to the $N$ (black dashed) and $M$ (red dotted) peaks in the two-phase region.

the sharp $M$ phase peak yields a correlation length of $\sim 600$ $\AA$ ( $\sim 18$ columns). The temperature dependences of $d$ and $\xi_{\perp}$ are shown in Fig. 7(a). On cooling, the observed phase sequence $I \rightarrow(I+M) \rightarrow(N+M) \rightarrow N$ is consistent with the phase diagram in Fig. 2(a) except that the pure $M$ phase was not obtained even at room temperature.

The behavior of the $25 \mathrm{wt} \%$ sample is similar to the $20 \mathrm{wt} \%$ sample as evident from the temperature dependence of $d$ and $\xi_{\perp}$ in Fig. 7(b). The $N$ phase appears at lower temperatures instead of the $M$ phase. This is a significant but not surprising deviation from the schematic phase diagram in Fig. 2(a). This suggests that for our samples, the $N$ region extends to higher concentrations than shown in Fig. 2(a), most likely due to different sample purity and concentration as mentioned above.
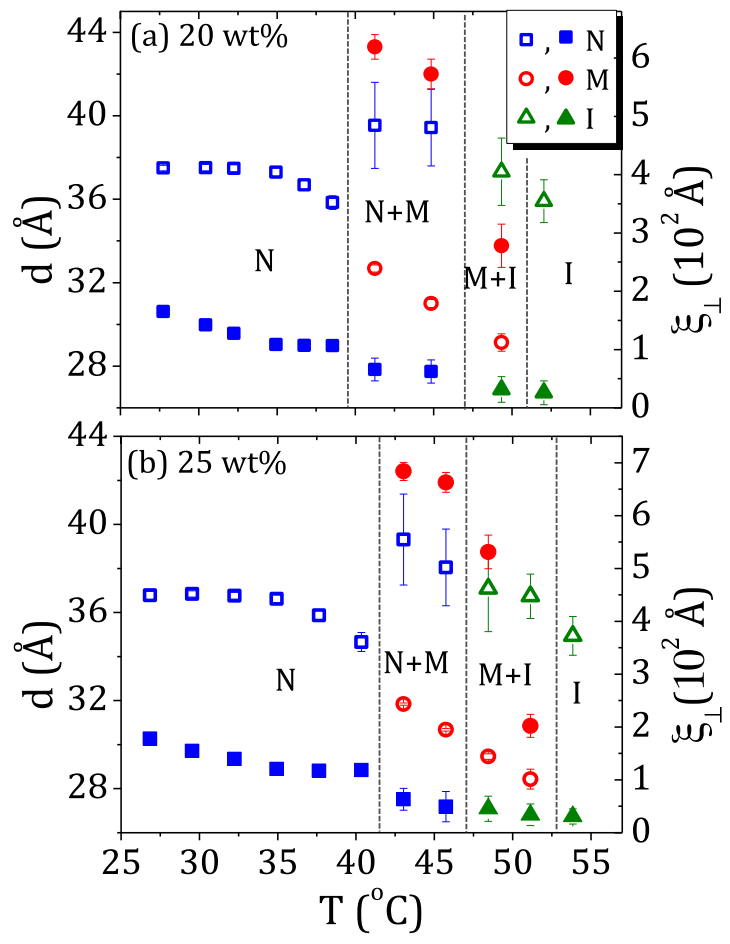

FIG. 7. (Color online) Temperature dependences of $d$ spacing (open symbols, left ordinate) and $\xi_{\perp}$ (filled symbols, right ordinate) for (a) $20 \mathrm{wt} \%$ and (b) $25 \mathrm{wt} \%$ DSCG aqueous solution in the $N$, $M$, and $I$ phases. 
The 25 wt $\%$ sample was very viscous which made capillary filling very difficult. The salient point from x-ray data is the appearance of the $M+I$ above the $N+M$ phase. This finding suggests the need for a careful reexamination of the phase diagram of the DSCG-water system, which is beyond the scope of this work. Since the transitions between the three phases of this system are first order, the observed phase boundaries depend on the direction and the rate of change of temperature as well as the experimental technique used. The optical texture observations are performed in a much shorter time than x-ray measurements leading to different transition temperatures.

In the $M$ phase, the diameter $d_{s}$ of the columnar stacks is estimated assuming that the right-circular unimolecular cylindrical DSCG columns are completely separate from water using [25]

$$
d_{s}=d_{p}(2 \sqrt{3} \phi / \pi)^{1 / 2}
$$

where $d_{p}=2 d_{1} / \sqrt{3}$ is the distance between the assemblies, and $\varphi$ is the volume fraction $[8,26]$ of DSCG. A value of $\rho=$ $1.55 \times 10^{3} \mathrm{~kg} / \mathrm{m}^{3}$ for the density of pure DSCG was used to find the volume fraction [26]. The value of $d_{s}$ varies from 16 to $19 \AA$, in agreement with both its previous estimate [8] of $16 \AA$ and of the column area [27] of $2.4 \mathrm{~nm}^{2}$. This rules out the chimneylike structure with its much larger dimensions as already retracted by Lydon [10].

Finally, the line shape of the $3.4 \AA$ peak is analyzed to calculate $\xi_{\|}$over which the DSCG molecules within a column are positionally correlated in the direction of its long axis. The temperature dependence of $\xi_{\|}$and $d$ for all concentrations is shown in Fig. 8. In the $N$ phase, the average length of assemblies is $\sim 80 \AA$, or 23 molecules. In the $N+M$ or $I+M$
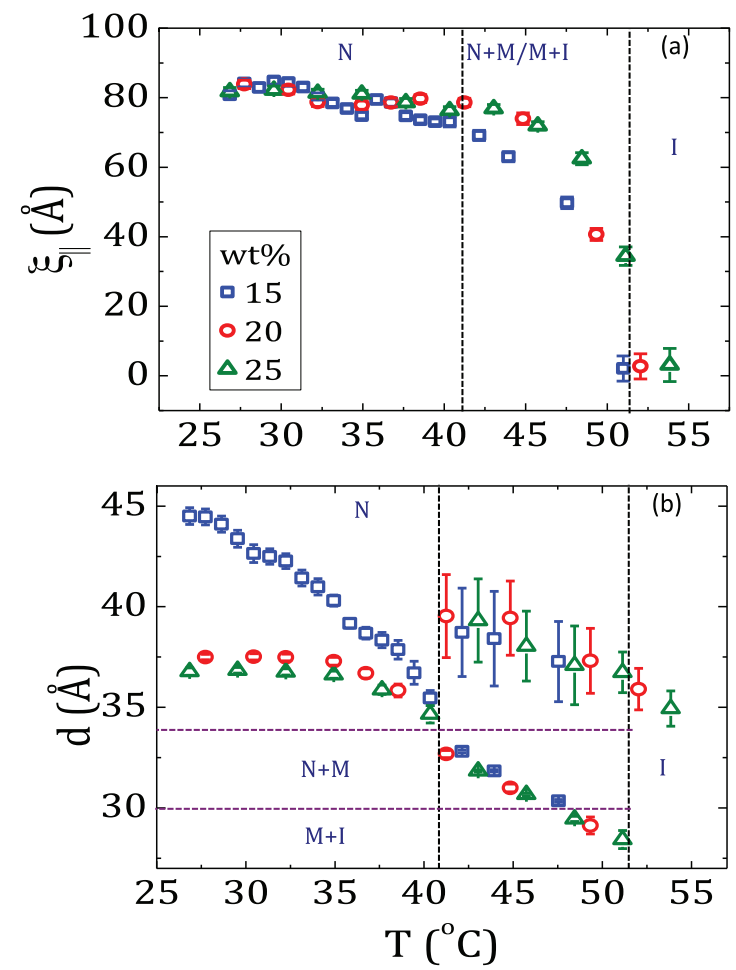

FIG. 8. (Color online) Temperature dependence of (a) assembly length $\xi_{\|}$and (b) interassembly distance $d$ for all solutions: $15 \mathrm{wt} \%$ (blue squares), $20 \mathrm{wt} \%$ (red circles), and $25 \mathrm{wt} \%$ (green triangles). biphasic regions, they become shorter and $\xi_{||}$decreases to $40 \AA$, i.e., $\sim 12$ molecules.

If the assemblies were threadlike [20], then the DSCG threads will be oriented parallel to the magnetic field due to the diamagnetic anisotropy of the DSCG molecules. The plane of the aromatic parts of the molecule will have orientational degeneracy with respect to the field direction. Consequently, (i) the first peak, corresponding to the length [16 $\AA$, Fig. 1(a)] of the molecules, would appear at small angles parallel to the magnetic field; (ii) this peak would be concentration independent and relatively sharp as the positional correlations $\left(\xi_{\perp}\right)$ would extend over the entire length of the thread; (iii) the second peak corresponding to the width of the molecule $(\sim 11 \AA)$ would be observed in the direction perpendicular to the field; (iv) the $d$ corresponding to the second peak would be concentration and temperature dependent; (v) it would be relatively broad due to the lack of positional correlations; and (vi) the $3.4 \AA$ peak perpendicular to the magnetic field would be concentration independent and relatively broad as the positional correlations $\left(\xi_{\|}\right)$transverse to the threads would be short range and extend to only two to three molecules according to the model. Clearly, the experimental results reported here contradict these expectations and strongly suggest that this model is an unlikely candidate for the DSCG assembly structure.

Temperature dependence of the interassembly distance $d$ for all mixtures clearly shows [Fig. 8(b)] that the assemblies are farther apart in the $N$ phase than in the regions of the $M$ phase. The separation between assemblies in the $N$ phase decreases as the number of stacks increases with increasing concentration. The plot in Fig. 8 further shows that, for the three concentrations above $\sim 40{ }^{\circ} \mathrm{C}, d$ corresponding to the sharp $M$ phase peak in the biphasic region becomes independent of the concentration and is $<34 \AA$ irrespective of the initial concentration. In this region, the values of $d$ calculated from the $N$ and $I$ peaks also are of approximately equal value. This is possible only if the samples undergo phase separation (spinodal decomposition) into the DSCG-rich and water-rich regions, with each region exhibiting a different phase. This process allows for regions of the $M$ phase to appear

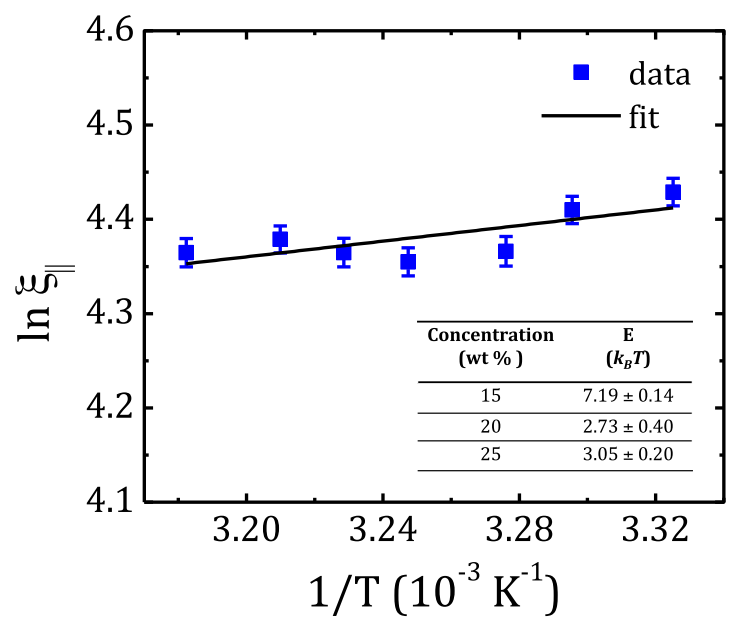

FIG. 9. (Color online) Calculation of the scission energy $E$ in the $N$ phase for $20 \mathrm{wt} \%$. Values of the scission energy for all mixtures are shown in the table (inset). The scission energy is determined from the slope of the straight line fit. 
even in low-concentration samples above the $N$ phase as has been previously reported [13] based on optical textures. As the temperature increases above $\sim 51{ }^{\circ} \mathrm{C}$, these coexisting phases coalesce into a single $I$ phase [Fig. 8]. In the $I$ phase region, the values of $d$ are comparable to the $N$ phase but the correlation lengths become smaller and comparable to the thickness of the DSCG molecule, i.e., the assemblies are very short.

Assuming the temperature dependence of assembly length to be Arrhenius $[17,28], \xi_{\|}=\xi_{0} \exp \left(\frac{E}{2 k_{B} T}\right)$, where $k_{B}$ is the Boltzmann's constant and $\xi_{0}$ is the bare correlation length, the scission or activation energy $E$ (i.e., energy required to remove one molecule from the assembly) can be estimated from the slope of the linear fit [Fig. 9]. A value of $E=6.8$ $\pm 0.8 k_{B} T$ for DSCG has been previously reported [27], but for concentrations more than an order of magnitude lower than used in this study. The scission energy in the $N$ phase is clearly concentration dependent and listed in the inset of Fig. 9. This is in contradiction to the assumption [29,30] that the aggregation process in DSCG is isodesmic. That assumption was based on experiments done at concentrations far below those investigated here.

To summarize, the results reported here make a compelling case that DSCG molecular assemblies are interacting single- molecule assemblies of stacked molecules. The distance between the assemblies and the assembly length are generally temperature and concentration dependent. These data are also inconsistent with the threadlike assembly suggested by $\mathrm{Wu}$ et al. [20]. There is no direct or indirect evidence supporting the chimneylike model. The counterintuitive presence of the regions of the $M$ phase above the $N$ phase and the coexistence of the $M$ and $I$ phases in all samples are found to occur via spinodal decomposition into water-rich and DSCG-rich regions.

The authors thank the Basic Energy Sciences program of the Office of Science, Department of Energy (DOE), for financial support under Grant No. DE-SC0001412. Use of the National Synchrotron Light Source, Brookhaven National Laboratory, was supported by the Basic Energy Sciences program of the DOE's Office of Science under Contract No. DE-AC02-98CH10886. Support by the European Synchrotron Research Facility (ESRF, Beamline ID02, Experiment No. HD-538) and the help by Manuel Fernandez-Martinez and Theyencheri Narayanan are gratefully acknowledged. We also thank Oleg Lavrentovich for providing high purity DSCG and Edgar Kooijman for the TLC work to confirm sample purity.
[1] J. S. Cox, G. D. Woodard, and W. C. McCrone, J. Pharm. Sci. 60, 1458 (1971).

[2] R. E. C. Altounyan, Schweiz. Med. Wochenschr. 110, 179 (1980); J. Pepys and A. E. Frankland, Disodium Cromoglycate in Allergic Airways Disease (Butterworths, London, 1970).

[3] R. M. Wood, Analytical Methods for Food Additives (CRC Press, Boca Raton, FL, 2004).

[4] K. Ichimura, T. Fujiwara, M. Momose, and D. J. Matsunaga, J. Mater. Chem. 12, 3380 (2002); K. Ichimura, M. Momose, K. Kudo, H. Akiyama, and N. Ishizuki, Langmuir 11, 2341 (1995); D. Matsunaga, T. Tamaki, H. Akiyama, and K. Ichimura, Adv. Mater. 14, 1477 (2002).

[5] A. Tolkki, E. Vuorimaa, V. Chukharev, H. Lemmetyinen, P. Ihalainen, J. Peltonen, V. Dehm, and F. Würthner, Langmuir 26, 6630 (2010); S. K. Lee, Y. Zu, A. Herrmann, Y. Geerts, K. Müllen, and A. J. Bard, J. Am. Chem. Soc. 121, 3513 (1999).

[6] K. Müllen and U. Scherf, Organic Light Emitting Devices: Synthesis, Properties and Applications (Wiley-VCH, Weinheim, 2006).

[7] S.V. Shiyanovskii, O.D. Lavrentovich, T. Schneider, T. Ishikawa, I. I. Smalyukh, C. J. Woolverton, G. D. Niehaus, and J. W. Doane, Mol. Cryst. Liq. Cryst. 434, 259/[587] (2005).

[8] S. Hamodrakas, A. J. Geddes, and B. Sheldrick, J. Pharm. Pharmacol. 26, 54 (1974).

[9] N. H. Hartshorne and G. D. Woodard, Mol. Cryst. Liq. Cryst. 23, 343 (1973).

[10] J. E. Lydon, Mol. Cryst. Liq. Cryst. Lett. 64, 19 (1980).

[11] T. K. Attwood and J. E. Lydon, Mol. Cryst. Liq. Cryst. 108, 349 (1984).

[12] D. Goldfarb, Z. Luz, N. Spielberg, and H. Zimmerman, Mol. Cryst. Liq. Cryst. 126, 225 (1985).

[13] G. J. T. Tiddy, D. L. Mateer, A. P. Ormerod, W. J. Harrison, and D. J. Edwards, Langmuir 11, 390 (1995).

[14] W. J. Harrison, D. L. Mateer, and G. J. T. Tiddy, J. Phys. Chem. 100, 2310 (1996).
[15] C. Ruslim, D. Matsunaga, M. Hashimoto, T. Takashi, and K. Ichimura, Langmuir 19, 3686 (2003).

[16] V. R. Horowitz, L. A. Janowitz, A. L. Modic, P. A. Heiney, and P. J. Collings, Phys. Rev. E 72, 041710 (2005).

[17] Y. A. Nastishin, H. Liu, T. Schneider, V. Nazarenko, R. Vasyuta, S. V. Shiyanovskii, and O. D. Lavrentovich, Phys. Rev. E 72, 041711 (2005).

[18] J. Lydon, J. Mater. Chem. 20, 10071 (2010); Liq. Cryst. 38, 1663 (2011).

[19] P. J. Collings, A. J. Dickinson, and E. C. Smith, Liq. Cryst. 37, 701 (2010).

[20] L. Wu, J. Lal, K. Simon, E. Burton, and Y.-Y. Luk, J. Am. Chem. Soc 131, 7430 (2009).

[21] L. Tortora, H.-S. Park, S.-W. Kang, V. Savaryn, S.-H. Hong, K. Kaznatcheev, D. Finotello, S. Sprunt, S. Kumar, and O. D. Lavrentovich, Soft Matter 6, 4157 (2010).

[22] A. P. Hammersley, S. O. Svensson, M. Hanfland, A. N. Fitch, and D. Hausermann, High Press. Res. 14, 235 (1996).

[23] A. Lorenz, N. Zimmermann, S. Kumar, D. R. Evans, G. Cook, M. Fernández Martínez, and H.-S. Kitzerow, J. Phys. Chem. B 117, 937 (2013).

[24] A. Lorenz, N. Zimmermann, S. Kumar, D. R. Evans, G. Cook, and H.-S. Kitzerow, Appl. Opt. 52, E1 (2013).

[25] V. Luzzati, Biological Membranes (Academic Press, New York, 1968), p. 78

[26] H.-S. Park, Ph.D. Dissertation, Kent State University, 2010.

[27] A. J. Dickinson, N. D. LaRacuente, C. B. McKitterick, and P. J. Collings, Mol. Cryst. Liq. Cryst. 509, 9/[751] (2009).

[28] Y. A. Nastishin, H. Liu, S. V. Shiyanovskii, O. D. Lavrentovich, A. F. Kostko, and M. A. Anisimov, Phys. Rev. E 70, 051706 (2004).

[29] M. R. Tomasik and P. J. Collings, J. Phys. Chem. B 112, 9883 (2008).

[30] L. Joshi, S.-W. Kang, D. M. Agra-Kooijman, and S. Kumar, Phys Rev E 80, 041703 (2009). 